\title{
THE MINIMAL STAGE, ENERGY PRESERVING RUNGE-KUTTA METHOD FOR POLYNOMIAL HAMILTONIAN SYSTEMS IS THE AVERAGED VECTOR FIELD METHOD
}

\author{
ELENA CELLEDONI, BRYNJULF OWREN, AND YAJUAN SUN
}

\begin{abstract}
No Runge-Kutta method can be energy preserving for all Hamiltonian systems. But for problems in which the Hamiltonian is a polynomial, the averaged vector field (AVF) method can be interpreted as a Runge-Kutta method whose weights $b_{i}$ and abscissae $c_{i}$ represent a quadrature rule of degree at least that of the Hamiltonian. We prove that when the number of stages is minimal, the Runge-Kutta scheme must in fact be identical to the AVF scheme.
\end{abstract}

\section{INTRODUCTION AND MAIN RESULT}

We shall be concerned with canonical Hamiltonian systems

$$
y^{\prime}=J^{-1} \nabla H(y)=f(y), \quad J=\left(\begin{array}{cc}
0 & I \\
-I & 0
\end{array}\right) .
$$

The numerical solution of problems of this type has been treated extensively in the literature; we refer to the monographs [8,11] and the references therein for details. Two of the most important properties of the system (1.1) are that the flow is a symplectic map and the Hamiltonian $H(y)$ is preserved along any solution $y(t)$. The circumstances under which various numerical integrators inherit these properties are by now fairly well understood. It is well known, for example, that B-series methods applied to (1.1) cannot simultaneously be symplectic and preserve the Hamiltonian [5] ; also, see [15] for a different and earlier version of the same result regarding one-step methods. We use the term $B$-series method for any computable integration method which has a B-series [8], i.e., a power series in the time step in which each term is a sum of elementary differentials of the vector field. An important advantage of B-series methods is that they preserve linear invariants and affine symmetries automatically. This is not the case, for example, for splitting methods and projection methods.

There has been a fair amount of work in the last two decades studying Bseries having certain geometric properties: symplectic B-series; energy/integralpreserving B-series; conjugate-to-symplectic B-series, and the simultaneous preservation of two or more of these properties [1, [12, [6], [5, [3, 9]. The goal of this endeavor is a complete characterization of B-series maps and their associated vector fields satisfying the different geometric features, and the study of their algebraic properties. A practical outcome of this effort is a number of results and useful

Received by the editor November 5, 2012.

2010 Mathematics Subject Classification. Primary 65P10, 65L05; Secondary 65L06, 37M99.

Key words and phrases. Energy preservation, Runge-Kutta methods, polynomial Hamiltonian systems.

(C)2014 American Mathematical Society Reverts to public domain 28 years from publication 
no-go theorems serving as guidelines for the construction of geometric numerical integration methods.

The results of the present paper contribute to this effort. In this paper we focus on the preservation of the Hamiltonian itself, we study integrators generating a sequence of approximations $\left\{y_{n}\right\}$ to the solution of (1.1) such that $H\left(y_{n}\right)=H\left(y_{0}\right)$ for all $n \geq 1$.

It has been shown that, besides the exact solution of (1.1), there exist energy preserving B-series, but no Runge-Kutta method can be energy preserving for all Hamiltonian systems (1.1). In fact, it was pointed out in [2] that any energy preserving integrator for (1.1) must obey all quadrature conditions, thus ruling out all Runge-Kutta schemes. On the other hand, it was noted in [14] that the (AVF) method, defined as

$$
y_{n+1}=y_{n}+h \int_{0}^{1} f\left((1-\xi) y_{n}+\xi y_{n+1}\right) \mathrm{d} \xi
$$

preserves the Hamiltonian for all problems of the form (1.1). The AVF method has second order convergence and is a B-series method. In this case energy preservation is achieved by introducing the exact computation of an integral as a part of the method.

A rather different point of view can be obtained by restricting the class of problems (1.1) from general Hamiltonian functions to polynomial Hamiltonians and thus polynomial Hamiltonian vector fields. Then, geometric properties generally become easier to preserve; see, for example, [13] for numerous interesting examples in the case of quadratic vector fields.

In this paper we adopt this point of view and we consider what can be achieved when the Hamiltonian is polynomial and the integrator is a Runge-Kutta method. For linear Hamiltonians, the resulting ODE is constant and any consistent RungeKutta scheme will reproduce the exact solution. If the Hamiltonian is quadratic, then the resulting ODE is linear, and the condition for preserving energy is that the stability function of the method satisfies $R(z) R(-z)=1$. For polynomials of higher order it is not known to which extent Runge-Kutta methods can preserve the Hamiltonian.

In particular, when the Hamiltonian is a polynomial, the integral in (1.2) can be exactly resolved a priori, the same result is obtained if the integral in (1.2) is replaced by a quadrature rule of sufficiently high order. This was observed in [2]. In fact, a standard linear quadrature formula with abscissae $c=\left(c_{1}, \ldots, c_{s}\right)^{T}$ and weights $b=\left(b_{1}, \ldots, b_{s}\right)^{T}$, results in a Runge-Kutta method in which the Butcher matrix is given as $A=c b^{T}$. This immediately shows that for any polynomial Hamiltonian system, there exist Runge-Kutta methods which exactly preserve the energy. Note also that any choice of quadrature rule of sufficiently high order yields the same approximation, the AVF method is reproduced exactly.

As pointed out in [2] any energy-preserving integrator for (1.1) must obey all quadrature conditions, but for polynomial systems this can be relaxed. Letting the Hamiltonian be a polynomial of degree $m$, a necessary condition for the energy to be preserved is that the quadrature conditions hold up to order $m$, or in terms of Runge-Kutta coefficients

$$
\sum_{i} b_{i} c_{i}^{k-1}=\frac{1}{k}, \quad k=1, \ldots, m .
$$


Thus, in considering energy preserving Runge-Kutta methods for polynomial Hamiltonians of degree $\leq m$ one may immediately restrict the focus to schemes whose coefficients satisfy (1.3). If $m=2 s$, then the smallest possible number of stages in the scheme is $s$, the resulting abscissae and weights are those of the Gauss-Legendre quadrature rule. If $m=2 s-1$, then the smallest possible number of stages is still $s$, but the quadrature rule is not uniquely given, although applying the corresponding Runge-Kutta method with $A=c b^{T}$ yields the same result for all $\left(c_{i}, b_{i}\right)$ satisfying (1.3) for all $k \leq 2 s-1$. In such a situation, we are interested in answering the question of whether the Butcher matrix $A=c b^{T}$ is unique. We shall restrict our search to Butcher matrices satisfying the usual condition

$$
\sum_{j=1}^{s} a_{i j}=c_{i}, \quad i=1, \ldots, s .
$$

We shall prove the following theorem

Theorem 1.1. Let $m \geq 3$. Among all Runge-Kutta methods which exactly preserve all polynomial Hamiltonians of degree at most $m$, those with the minimal number of stages coincide with the AVF method (1.2) when applied to such problems. The number of stages in these methods is $\lfloor(m+1) / 2\rfloor$.

In general, there are energy preserving Runge-Kutta methods for polynomial Hamiltonian systems which do not coincide with the AVF-integrator. There also exist such methods of arbitrarily high order. Examples are easily obtained as composition methods based on the AVF-integrator, or by the collocation methods proposed in [10] and [7]. The technique we use to prove this result can be summarized as follows:

(1) The first step is to consider a set of conditions for energy preservation which are linear in the Butcher matrix $A$, these conditions are called the double bush conditions and may be thought of as a linear system $M(A)=w$ where $M$ is a linear map from $\mathbf{R}^{s \times s}$ into $\mathbf{R}^{d}$ for some $d$ to be specified.

(2) Then the rank of $M$ is determined, the results are different in the even $(m=2 s)$ and odd $(m=2 s-1)$ cases. A particular basis for the kernel of $M$ is identified in each of the cases.

(3) The discretized AVF method, $A=c b^{T}$, represents a known solution and any other solution must be of the form $A=c b^{T}+N$, where $N$ is in the kernel of $M$. We show, by using certain nonlinear energy preserving conditions that such solutions require $N=0$.

To prove results about energy preserving Runge-Kutta methods for polynomial Hamiltonian systems, we use selected B-series energy preserving conditions from [5] in a novel fashion. We expect that the techniques developed here can be used for studying other, similar, geometric features of integrators.

We list here some of the consequences arising from this result:

- Energy preserving Runge-Kutta methods with a minimal number of stages cannot have order higher than 2. In other words, if $H$ is a polynomial of degree $m \geq 3$ we need at least $\lfloor(m+1) / 2\rfloor$ to construct an energy preserving Runge-Kutta method of order 2, and to get higher order one needs to add more stages; this point of view was also adopted in [10. 
- Similarly, if $H$ is a polynomial of degree $m \geq 3$ we need at least $\lfloor(m+1) / 2\rfloor$ stages to construct an energy preserving Runge-Kutta method of order 2 which is conjugate to symplectic up to order 4 (see [2]).

The rest of the paper is organized as follows: In Section 2 we review some tools needed from the literature, and we provide general conditions for energy preservation of B-series methods needed in the proof. In Section 3, we prove Theorem 1.1 for the case of even polynomial degree of the Hamiltonian, the proof for the odd case is given in [4].

\section{ENERGy PRESERVATION FOR B-SERIES METHOdS}

For an introduction to B-series and the notation we use for rooted trees and free trees, see for instance [8]. We remind the reader that a nonsuperfluous free tree is a free tree with the property that the deletion of any single edge results in two nonidentical trees. A corner stone in our approach is the following result from 5 .

Theorem 2.1. A map with B-series $B(a, y)$ preserves energy up to order $n$ if and only if

$$
\sum_{u \in \pi^{-1}(\bar{t})} \frac{(-1)^{\kappa(t, u)}}{\sigma(u)} a\left(B_{-}(u)\right)=0, \quad \forall \bar{t} \in \bigcup_{k \leq n+1} F T^{k} .
$$

Here $t$ is a designated member of the equivalence class (free tree) $\bar{t}$. $F T^{k}$ is the set of nonsuperfluous free trees with precisely $k$ vertices, $\kappa(t, u)$ is the number of root shifts needed to obtain the rooted tree $u$ from the rooted tree $t, \sigma(t)$ is the symmetry coefficient of the rooted tree $t$. The notation $a\left(B_{-}(u)\right)$ signifies the product $a\left(u_{1}\right) \cdots a\left(u_{r}\right)$ where $u_{1}, \ldots, u_{r}$ are the subtrees of $u$.

Readers who are unfamiliar with the B-series approach should still be able to follow our discussion, since the results of Chartier et al. [5] are only used to write down conditions on the coefficients of the Runge-Kutta method which are necessary for energy preservation. A self-contained version of the present paper can also be found in [4].

One may remark that all the conditions of the theorem must be satisfied in order for the corresponding method to be energy preserving for every Hamiltonian function $H$. However, in this article we are interested in the subclass of Hamiltonians which are multivariate polynomials of some prescribed degree $m$. In fact, one finds that any nonsuperfluous free tree having a vertex with more than $m$ emanating branches causes (2.1) to be a trivial statement $(0=0)$. Conversely, one may verify that conditions corresponding to nonsuperfluous free trees with at most $m$ emanating branches from any vertex from a linearly independent set when considered over all Hamiltonians of degree at most $m$.

The following result is inspired by 2 .

Theorem 2.2. Any consistent B-series method with coefficients a $(t)$ which is energy preserving for all polynomial Hamiltonians of degree $m$ satisfies the quadrature conditions of order $k$ for $1 \leq k \leq m$, i.e.,

$$
a\left(\left[\bullet^{k-1}\right]\right)=\frac{1}{k}, \quad 1 \leq k \leq m .
$$


If the method is a Runge-Kutta method with abscissae $c_{i}$ and weights $b_{i}, i=1, \ldots, s$ then a necessary condition for energy preservation is

$$
\sum_{i=1}^{s} b_{i} c_{i}^{k-1}=\frac{1}{k}, \quad k=1, \ldots, m .
$$

Proof. For $1 \leq k \leq m$ we consider (2.1) for the free tree with $k+1$ vertices containing the bushy tree $t=\left[\bullet^{k}\right]$ (i.e., the tree consisting of $k$ copies of the onenode tree as subtrees). There is only one other tree in the equivalence class, namely $\left.t^{\prime}=\left[\bullet^{k-1}\right]\right]$. Now $a\left(B_{-}(t)\right)=a\left(\bullet^{k}\right)=a(\bullet)^{k}=1$ for any consistent method. On the other hand, $a\left(B_{-}\left(t^{\prime}\right)\right)=a\left([\bullet]^{k-1}\right)$ and together with (2.1) and the fact that $\kappa\left(t, t^{\prime}\right)=1, \sigma(t)=k$ ! and $\sigma\left(t^{\prime}\right)=(k-1)$ ! we get the desired result. For RungeKutta methods it is well known that the $a\left(\left[\bullet^{k-1}\right]\right)$ is the left-hand side of $(2.2)$.

2.1. The double bush conditions. A certain subset of the nonsuperfluous free trees will play a particular role here, these are the trees which yield linear conditions on the matrix $A$. We consider the double bush free trees that we denote $t_{p, q}$ for integers $p$ and $q$ in $\{1,2, \ldots, m-1\}$. Clearly $t_{p, p}$ is superfluous, and by symmetry, $t_{p, q}=t_{q, p}$, so one will typically require $1 \leq p<q \leq m-1$.

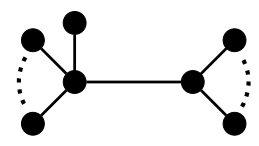

Figure 1. The double bush free tree $t_{p, q}$ having $p$ leaves on one side and $q$ on the other

For $q=m-1$ the maximal number of branches from a vertex is $m$. We state the resulting conditions for energy preservation in the following lemma.

Lemma 2.3. Let $(A, b, c)$ be a Runge-Kutta scheme whose abscissae and weights satisfy the quadrature conditions (2.2) for $1 \leq k \leq m$. Then the conditions for energy preservation imposed on the method by the double bush free trees $t_{p, q}$, henceforth called the double bush conditions are

$$
p b^{T} C^{p-1} A c^{q}-q b^{T} C^{q-1} A c^{p}=\frac{1}{q+1}-\frac{1}{p+1}, \quad 1 \leq p<q \leq m-1 .
$$

Here $c=\left(c_{1}, \ldots, c_{s}\right)^{T}, C=\operatorname{diag}\left(c_{1}, \ldots, c_{s}\right)$. Powers of $c$ are defined componentwise. A particular solution to the double bush conditions is given as

$$
A_{\mathrm{avf}}=c b^{T} .
$$

Proof. The free tree $t_{p, q}$ is the equivalence class containing the four distinct rooted trees: $t_{1}=\left[\left[\bullet^{p-1}\left[\bullet^{q}\right]\right]\right], t_{2}=\left[\bullet^{p}\left[\bullet^{q}\right]\right], t_{3}=\left[\bullet^{q}\left[\bullet^{p}\right]\right]$ and $t_{4}=\left[\left[\bullet^{q-1}\left[\bullet^{p}\right]\right]\right]$.

One then has $\kappa\left(t_{i}, t_{j}\right)=|i-j|$. By the quadrature conditions,

$$
a\left(B_{-}\left(t_{2}\right)\right)=a(\bullet)^{p} a\left(\left[\bullet^{q}\right]\right)=\frac{1}{q+1}, \quad a\left(B_{-}\left(t_{3}\right)\right)=a(\bullet)^{q} a\left(\left[\bullet^{p}\right]\right)=\frac{1}{p+1},
$$

whereas

$$
a\left(B_{-}\left(t_{1}\right)\right)=a\left(\left[\bullet^{p-1},\left[\bullet^{q}\right]\right]\right)=b^{T} C^{p-1} A c^{q}, \quad a\left(B_{-}\left(t_{4}\right)\right)=a\left(\left[\bullet^{q-1},\left[\bullet^{p}\right]\right]\right)=b^{T} C^{q-1} A c^{p} .
$$

We also compute $\sigma\left(t_{1}\right)=(p-1) ! q ! \sigma\left(t_{2}\right)=\sigma\left(t_{3}\right)=p ! q !$, and $\sigma\left(t_{4}\right)=p !(q-1)$ !. Substituting all this into the conditions (2.1) we get the stated conditions. 

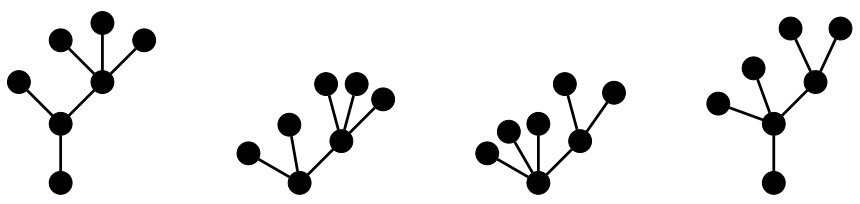

Figure 2. The four trees in the double bush equivalence class $t_{2,3}$

We finally substitute (2.4) for $A$ in (2.3) and use the quadrature conditions to get

$p b^{T} C^{p-1} c b^{T} c^{q}-q b^{T} C^{q-1} c b^{T} c^{p}=\frac{p}{(p+1)(q+1)}-\frac{q}{(q+1)(p+1)}=\frac{1}{q+1}-\frac{1}{p+1}$.

For a given $s$, the double bush conditions (2.3) define a linear operator $M: \mathbf{R}^{s \times s} \rightarrow$ $\mathbf{R}^{\frac{1}{2}(m-1)(m-2)}$ acting on the set of Butcher matrices $A$. Generally, this operator depends on the quadrature coefficients $\left(b_{i}, c_{i}\right)$, as well as on $m$ and $s$. However, in our case we shall always be concerned with quadrature formulas of the highest possible order, so that we have either $m=2 s$ or $m=2 s-1$. We can then also represent the abscissae and weights of the quadrature formula by means of a single real parameter $\zeta$ as follows: We assume that $\left(c_{1}, \ldots, c_{s}\right)$ are the distinct zeros of the polynomial $P_{s}(x)-\zeta P_{s-1}(x)$ where $P_{q}$ is the $q$ th degree Legendre polynomial relative to the interval $[0,1]$, and the weights are determined by solving (1.3) for $k \leq s$. We would thus have $M=M(\zeta, s, m)$, but we shall restrict our attention to the case for even $m, M=M(0, s, 2 s)$, while the odd case $M(\zeta, s, 2 s-1)$ is reported in [4]. The following lemma is included without proof for future reference

Lemma 2.4. In both the even and odd cases, the matrix $N_{1}=(\mathbf{1}-c) b^{T}$ is in the kernel of $M$, i.e., $M\left((\mathbf{1}-c) b^{T}\right)=0$, where $\mathbf{1}=(1, \ldots, 1)^{T} \in \mathbf{R}^{s}$.

It is useful to combine the double bush conditions into conditions involving arbitrary polynomials in $C$ and $c$ rather than the monomials used in the previous lemma, we shall let $\Pi_{p}$ denote the linear space of polynomials of degree at most $p$.

Lemma 2.5. Let $(A, b, c)$ be a Runge-Kutta scheme whose abscissae and weights satisfy the quadrature conditions (2.2) for $1 \leq k \leq m$. Assume that it also satisfies the double bush conditions (2.3). Let $P \in \Pi_{p}$ and $Q \in \Pi_{q}$ such that $P(0)=Q(0)=$ 0 . Then

$$
b^{T} P^{\prime}(C) A Q(c)-b^{T} Q^{\prime}(C) A P(c)=P(1) \int_{0}^{1} Q(t) \mathrm{d} t-Q(1) \int_{0}^{1} P(t) \mathrm{d} t .
$$

Proof. Write $P(z)=\sum_{p^{\prime}} \alpha_{p^{\prime}} z^{p^{\prime}}$ and $Q(z)=\sum_{q^{\prime}} \beta_{q^{\prime}} z^{q^{\prime}}$. Then, using Lemma 2.3.

$$
\begin{array}{r}
b^{T} P^{\prime}(C) A Q(c)-b^{T} Q^{\prime}(C) A P(c)=\sum_{p^{\prime}, q^{\prime}} \alpha_{p^{\prime}} \beta_{q^{\prime}}\left(p^{\prime} b^{T} C^{p^{\prime}-1} A c^{q^{\prime}}-q^{\prime} b^{T} C^{q^{\prime}-1} A c^{p^{\prime}}\right) \\
=\sum_{p^{\prime}, q^{\prime}} \alpha_{p^{\prime}} \beta_{q^{\prime}}\left(\frac{1}{q^{\prime}+1}-\frac{1}{p^{\prime}+1}\right)=P(1) \int_{0}^{1} Q(t) \mathrm{d} t-Q(1) \int_{0}^{1} P(t) \mathrm{d} t .
\end{array}
$$


2.2. The case $s=2, m=3$. The general ideas of the proof can be illustrated for the case where a two stage Runge-Kutta method is applied to problems with a cubic Hamiltonian. Then the abscissae, $c_{1}, c_{2}$ must be those of a third order quadrature rule. Thus, they are the zeros of $P_{2}(x)-\zeta P_{1}(x)$ for some $\zeta \in \mathbf{R}$, or equivalently the abscissae satisfy the condition $3\left(c_{1}+c_{2}\right)-6 c_{1} c_{2}=2$. Another consequence of the quadrature conditions is that $b^{T} c^{3}=\frac{1}{4}+\frac{1}{36} \zeta$.

There is just one double bush condition (2.3) with $p=1, q=2$, we therefore consider

$$
b^{T} N c^{2}-2 b^{T} C N c=0
$$

for the elements of the kernel of the operator $M$. We represent $N$ in the form

$$
N=\alpha_{1,1} \mathbf{1} b^{T}+\alpha_{2,1} c b^{T}+\alpha_{1,2} \mathbf{1} b^{T} C+\alpha_{2,2} c b^{T} C .
$$

By substituting this into (2.6), and using the quadrature conditions, we get

$$
\alpha_{11}+\alpha_{2,1}+\left(\frac{1}{2}-\frac{1}{6} \zeta\right) \alpha_{1,2}+\left(\frac{7}{12}-\frac{1}{12} \zeta\right) \alpha_{2,2}=0 .
$$

The solutions of (2.6) thus form a three dimensional space, a basis is given as

$$
N_{1}=(\mathbf{1}-c) b^{T}, \quad N_{2}=2 \mathbf{1} b^{T}+3(\mathbf{1}-2 c) b^{T} C, \quad N_{3}=2 \zeta \mathbf{1} b^{T}+3(7 \mathbf{1}-6 c) b^{T} C
$$

So any Butcher matrix candidate must be of the form $A=c b^{T}+N$ where $N=$ $v_{1} N_{1}+v_{2} N_{2}+v_{3} N_{3}$ and the row sum condition (1.4) then implies $N 1=0$. We obtain after some calculations that $N \in \operatorname{ker} M$ satisfying this condition is of the form

$$
N=\beta((\zeta-1) \mathbf{1}-2 \zeta c) b^{T}(I-2 C), \quad \beta \in \mathbf{R} .
$$

There are several possible nonlinear conditions to choose from in order to prove that any candidate solution of the form $A=c b^{T}+N$ would require $N=0$. We first use the the tall tree with 5 nodes, $\bar{t}=2.1$ in and apply the quadrature conditions up to third order as well as the identity $b^{T} c^{3}=\frac{1}{4}+\frac{1}{36} \zeta$. The condition (2.1) reduces to

$$
\frac{1}{18} \beta^{2} \zeta=0
$$

showing that if $\zeta \neq 0$, we get $\beta=0$ and we are left with the AVF scheme. To prove the same result for $\zeta=0$ which corresponds to the two-point Gauss-Legendre quadrature, we repeat the calculations using the free tree

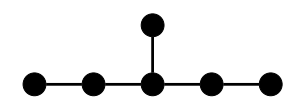

and we obtain the condition $\frac{1}{72} \beta^{2}=0$. This proves Theorem 1.1 in the case $m=3, s=2$.

\section{The CASE of EVEn DEgree Hamiltonians}

In this section, we prove Theorem 1.1 for the case that the polynomial Hamiltonian is of even degree $m=2 s$, such that the underlying quadrature is the GaussLegendre formula. We use the following notation for the standard $L^{2}$ inner product between functions $u$ and $v$,

$$
\langle u, v\rangle=\int_{0}^{1} u(x) v(x) \mathrm{d} x .
$$


For every nonnegative integer $q$, we let $P_{q}$ be the Legendre polynomial of degree $q$,

$$
P_{q}(x)=\frac{1}{q !} \frac{\mathrm{d}^{q}}{\mathrm{~d} x^{q}} x^{q}(x-1)^{q},
$$

relative to the interval $[0,1]$, scaled such that $P_{q}(1)=1$ for every $q$, and consequently,

$$
\left\langle P_{k}, P_{\ell}\right\rangle=\frac{\delta_{k \ell}}{2 k+1}
$$

The polynomials

$$
G_{q}(x)=\int_{0}^{x} P_{q-1}(t) \mathrm{d} t, \quad q \geq 1,
$$

have for $q \geq 2$ the abscissae of the Gauss-Lobatto quadrature as zeros, and

$$
G_{q}(x)=\frac{1}{2(2 q-1)}\left(P_{q}(x)-P_{q-2}(x)\right)=\frac{1}{q(q-1)} x(x-1) P_{q-1}^{\prime}(x) .
$$

The following biorthogonality relations will be useful:

$$
\left\langle G_{1}, P_{\ell}^{\prime}\right\rangle=1 \forall \ell \in \mathbb{N}, \quad\left\langle G_{q+1}, P_{\ell}^{\prime}\right\rangle=-\frac{\delta_{\ell q}}{2 q+1} \forall q \geq 2, \ell \in \mathbb{N} .
$$

For any quadrature formula, we define the discrete counterpart to the inner product above,

$$
\langle u, v\rangle_{D}=\sum_{i=1}^{s} b_{i} u\left(c_{i}\right) v\left(c_{i}\right),
$$

and by a slight abuse of language we shall call it the discrete inner product. If the quadrature formula has order $m$ and $P$ and $Q$ are polynomials such that $\operatorname{deg} P+$ $\operatorname{deg} Q \leq m-1$, then

$$
\langle P, Q\rangle_{D}=\langle P, Q\rangle
$$

The discrete inner product can still be computed even in cases where it differs from the continuous one. The following result which will be of subsequent use, facilitates this in the case where $\operatorname{deg} P+\operatorname{deg} Q=m$.

Lemma 3.1. Suppose that a quadrature rule with abscissae $\left(c_{1}, \ldots, c_{s}\right)$ is exact for all polynomials of degree at most $m-1$, where $s \leq m \leq 2 s$, and let $\rho_{s}=\prod_{i=1}^{s}\left(x-c_{i}\right)$. Let $\pi_{m}$ be a monic polynomial of degree $m$. Then

$$
\left\langle\pi_{m}, 1\right\rangle_{D}=\left\langle\pi_{m}, 1\right\rangle-\left\langle\rho_{s}, \theta_{m-s}\right\rangle
$$

for any monic polynomial $\theta_{m-s}$ of degree $m-s$.

Proof. Let $\delta_{m-1}=\pi_{m}-\rho_{s} \theta_{m-s} \in \Pi_{m-1}$ for an arbitrary monic polynomial $\theta_{m-s} \in$ $\Pi_{m-s}$. Then

$\left\langle\pi_{m}, 1\right\rangle_{D}=\left\langle\delta_{m-1}+\rho_{s} \theta_{m-s}, 1\right\rangle_{D}=\left\langle\delta_{m-1}, 1\right\rangle_{D}=\left\langle\delta_{m-1}, 1\right\rangle=\left\langle\pi_{m}, 1\right\rangle-\left\langle\rho_{s}, \theta_{m-s}\right\rangle$. 
We can apply this lemma to obtain the following discrete inner products when Gauss-Legendre quadrature is used:

$$
\begin{aligned}
\left\langle P_{2 s-r}, P_{r}\right\rangle_{D} & =-\frac{\gamma_{2 s-r} \gamma_{r}}{\gamma_{s}^{2}(2 s+1)}, \quad \gamma_{\ell}=\frac{(2 \ell) !}{\ell !^{2}}, \\
\left\langle G_{2 s-r+1}, P_{r}^{\prime}\right\rangle_{D} & =\frac{r}{2 s-r+1}\left\langle P_{2 s-r}, P_{r}\right\rangle_{D} .
\end{aligned}
$$

Here $\gamma_{\ell}$ is the leading coefficient of $P_{\ell}$.

In analyzing the rank of the linear operator $M$, it is useful to work with the transformed double bush conditions given in Lemma 2.5, equation (2.5). Generally, one may select any suitable set of polynomials so that the rank of $M$ is not reduced. In this section we shall make the choices $G_{p}$ and $G_{q}$ for $P$ and $Q$, where $1 \leq p<$ $q \leq m-1$. It will also be convenient to write the elements $A$ in terms of a basis as follows:

$$
A=\sum_{k=1}^{s} \sum_{\ell=1}^{s} \alpha_{k, \ell} A_{k, \ell}, \quad A_{k, \ell}=P_{k-1}(c) b^{T} P_{\ell}^{\prime}(C) .
$$

The resulting equations for the coefficients $\alpha_{k, \ell}$ when considering $M(A)=0$ are

$$
\begin{aligned}
& \sum_{k=1}^{s} \sum_{\ell=1}^{s} \alpha_{k, \ell}\left(\left\langle P_{p-1}, P_{k-1}\right\rangle_{D}\left\langle G_{q}, P_{\ell}^{\prime}\right\rangle_{D}-\left\langle P_{q-1}, P_{k-1}\right\rangle_{D}\left\langle G_{p}, P_{\ell}^{\prime}\right\rangle_{D}\right) \\
& \quad=0,1 \leq p<q \leq m-1 .
\end{aligned}
$$

We prove the following result, in which we recall that $N_{1}=(\mathbf{1}-c) b^{T}$.

Lemma 3.2. Suppose $m=2 s, s \geq 2$ and $c, b$ are the abscissae and weights of the Gauss-Legendre quadrature. Then $\operatorname{rank}(M)=s^{2}-1$ and $\operatorname{ker} M=\operatorname{span}\left\{N_{1}\right\}$.

Proof. By Lemma 2.4, clearly $\operatorname{rank}(M) \leq s^{2}-1$. Note that the kernel element $N_{1}$ can be written in the format (3.11) as $N_{1}=(\mathbf{1}-c) b^{T}=\frac{1}{4}\left(A_{1,1}-A_{1,2}\right)$. Therefore, it must be true that $\operatorname{rank}(M) \geq s^{2}-1$ if some subset of the conditions (3.12) together with $\alpha_{1,1}=0$ cause the remaining $\alpha_{k, \ell}$ to vanish. It is enough to consider just $s^{2}-1$ (linearly independent) conditions among the $(2 s-1)(s-1)$. We select the conditions corresponding to $1 \leq p<q \leq 2 s-1$, and such that $p+q \leq 2 s+1$. The case $s=4$ is reported in Figures 3 and 4 . The $(p, q)$-element of the matrix in Figure 3 corresponds to the condition in (3.12). The numbers refer to the ordering in which the conditions are used in the proof. The ones marked $\left(n_{a}, n_{b}\right)$ are used simultaneously. The corresponding ordering of the unknowns $\alpha_{k, l}$ is reported in Figure 4

We begin by applying condition $(p, s+1)$ to $A_{k \ell}$ for $1 \leq p \leq s$. Since (3.6) applies for all inner products, we get from (3.2) and (3.4) that

$$
\begin{gathered}
\left\langle P_{p-1}, P_{k-1}\right\rangle_{D}\left\langle P_{\ell}^{\prime}, G_{s+1}\right\rangle_{D}-\left\langle P_{s}, P_{k-1}\right\rangle_{D}\left\langle P_{\ell}^{\prime}, G_{p}\right\rangle_{D} \\
=-\frac{s}{(s+1)(2 s+1)(2 k+1)} \delta_{p k} \delta_{s \ell} .
\end{gathered}
$$

Thus, $\alpha_{p s}=0$ for $1 \leq p \leq s$. We shall proceed by induction. Suppose it is true that

$$
\alpha_{k \ell}=0, \quad k>i+2, \ell>i+1,
$$




$$
p \downarrow\left[\begin{array}{ccccccc}
0 & 10 & 9_{a} & 7_{a} & 4 & 7_{b} & 9_{b} \\
& 0 & 8_{a} & 6_{a} & 3 & 6_{b} & 8_{b} \\
& & 0 & 5_{a} & 2 & 5_{b} & - \\
& & & 0 & 1 & - & - \\
& & & & 0 & - & - \\
& & & & & 0 & - \\
& & & & & & 0
\end{array}\right]
$$

FiguRE 3. Ordering of the conditions $(p . q)$

$$
k \downarrow\left[\begin{array}{cccc}
0 & 9_{a} & 7_{a} & 4 \\
10 & 8_{a} & 6_{a} & 3 \\
9_{b} & 8_{b} & 5_{a} & 2 \\
7_{b} & 6_{b} & 5_{b} & 1
\end{array}\right]
$$

Figure 4. Ordering of $\alpha_{k, l}$

which is established for $i=s-2$. We now prove, by using conditions $(p, i+2)$ and $(p, 2 s-i)$ together with (3.13) that $\alpha_{k, \ell}=0$ for $k>i+1, \ell>i$. The first of these conditions applied to $A_{k \ell}$ yields

$$
\left\langle P_{p-1}, P_{k-1}\right\rangle_{D}\left\langle P_{\ell}^{\prime}, G_{i+2}\right\rangle_{D}-\left\langle P_{i+1}, P_{k-1}\right\rangle_{D}\left\langle P_{\ell}^{\prime}, G_{p}\right\rangle_{D}
$$

(3.6) applies for all inner products and we conclude, using (3.2) and (3.4), that condition $(p, i+2)$ implies, after multiplying both sides by $(2 p-1)(2 i+3)$

$$
-\alpha_{p, i+1}+\alpha_{i+2, p-1}=0, \quad p>1
$$

and for $p=1$, multiplying each side by $2 i+3$ we get

$$
-\alpha_{1, i+1}-\sum_{\ell=1}^{s} \alpha_{i+2, \ell}=0, \quad p=1 .
$$

We next consider condition $(p, 2 s-i)$ applied to $A_{k \ell}$ to get

$$
\left\langle P_{p-1}, P_{k-1}\right\rangle_{D}\left\langle P_{\ell}^{\prime}, G_{2 s-i}\right\rangle_{D}-\left\langle P_{2 s-i-1}, P_{k-1}\right\rangle_{D}\left\langle P_{\ell}^{\prime}, G_{p}\right\rangle_{D}
$$

We readily compute $\left\langle P_{p-1}, P_{k-1}\right\rangle_{D}=\frac{\delta_{k p}}{2 p-1}$ and $\left\langle P_{\ell}^{\prime}, G_{p}\right\rangle_{D}=\frac{\delta_{p, \ell+1}}{2 p-1}$ if $p>1$, and $\left\langle P_{\ell}^{\prime}, G_{1}\right\rangle_{D}=1$. For $\left\langle P_{\ell}^{\prime}, G_{2 s-i}\right\rangle_{D}$, (3.6) applies when $\ell \leq i$ causing it to vanish by (3.4). For $\ell=i+1$ the total degree equals $2 s$, and by (3.10)

$$
\left\langle P_{i+1}^{\prime}, G_{2 s-i}\right\rangle_{D}=\frac{i+1}{(2 s-i)}\left\langle P_{2 s-i-1}, P_{i+1}\right\rangle_{D} .
$$

Nonzero entries for $\ell>i+1$ can be ignored due to the induction hypothesis. Finally, for $k \leq i+1$, one has $\left\langle P_{2 s-i-1}, P_{k-1}\right\rangle_{D}=\left\langle P_{2 s-i-1}, P_{k-1}\right\rangle=0$. For $k=i+2$, we invoke (3.9) just to assert that $\left\langle P_{2 s-i-1}, P_{i+1}\right\rangle_{D} \neq 0$ so that this factor can be cancelled in the condition $(p, 2 s-i)$ and we get

$$
-\frac{i+1}{2 s-i} \alpha_{p, i+1}+\alpha_{i+2, p-1}=0, \quad p>1
$$


and

$$
-\frac{i+1}{2 s-i} \alpha_{1, i+1}-\sum_{\ell=1}^{s} \alpha_{i+2, \ell}=0, \quad p=1 .
$$

Combining (3.14) and (3.16) we get for $p>1$ the system

$$
\left[\begin{array}{cc}
-1 & 1 \\
-\frac{i+1}{2 s-i} & 1
\end{array}\right]\left[\begin{array}{c}
\alpha_{p, i+1} \\
\alpha_{i+2, p-1}
\end{array}\right]=0
$$

and thus

$$
\alpha_{p, i+1}=\alpha_{i+2, p-1}=0, p=2, \ldots, i+1 .
$$

The remaining indices to be dealt with in the induction step are $\alpha_{1, i+1}$ and $\alpha_{i+2, i+1}$, and for these we consider (3.15) and (3.17). We use that the only element in the $(i+2)$ th row $\left(\alpha_{i+2, \ell}\right)$ not yet found to be zero is $\alpha_{i+2, i+1}$ and so we obtain also in the case $p=1$ a nonsingular $2 \times 2$ system where the two unknowns must satisfy $\alpha_{1, i+1}=\alpha_{i+2, i+1}=0$. The induction step is completed. The induction proof ensures that all $\alpha_{k \ell}=0$ except possibly $\alpha_{1,1}$ and $\alpha_{2,1}$, but the former is zero by assumption. But the remaining unused condition $(p, q)=(1,2)$ applied to $A_{2,1}$ yields $-1 / 3$ and thus also $\alpha_{2,1}=0$. In summary, we have proved that for an $s \times s$-matrix $A$ is expressed in the form (3.11) with $\alpha_{1,1}=0$, the conditions (3.12) imply $A=0$ which is equivalent to $\operatorname{rank}(M) \geq s^{2}-1$. Combined with the known null-vector $N_{1}=(\mathbf{1}-c) b^{T}$ this proves that the rank of $A$ is precisely $s^{2}-1$.

Proof of Theorem 1.1 (even case). From Lemma 2.3, (2.4) and Lemma 3.2 we know that any solution to the double bush conditions for $m=2 s \geq 3$ must be of the form $A=c b^{T}+\beta(\mathbf{1}-c) b^{T}$. But then the condition (1.4) immediately implies that

$$
A \mathbf{1}=c b^{T} \mathbf{1}+\beta(1-c) b^{T} \mathbf{1} \quad \Rightarrow \quad \beta(\mathbf{1}-c)=0
$$

so that $\beta=0$ and we are left with the AVF method.

The case of odd degree, $m \geq 5$ is similar although more technical, but follows the same method outlined in the introduction and exemplified in Subsection 2.2. A complete proof is given in 4 .

\section{ACKNOWLEDGMents}

This research was supported by a Marie Curie International Research Staff Exchange Scheme Fellowship within the 7th European Community Framework Programme, part of the work was carried out while the authors were visiting Massey University, Palmerston North, New Zealand and La Trobe University, Melbourne, Australia.

\section{REFERENCES}

[1] M. P. Calvo and J. M. Sanz-Serna, Canonical B-series, Numer. Math. 67 (1994), no. 2, 161-175, DOI 10.1007/s002110050022. MR1262779(95e:65072)

[2] Elena Celledoni, Robert I. McLachlan, David I. McLaren, Brynjulf Owren, G. Reinout W. Quispel, and William M. Wright, Energy-preserving Runge-Kutta methods, M2AN Math. Model. Numer. Anal. 43 (2009), no. 4, 645-649, DOI 10.1051/m2an/2009020. MR2542869 (2010i:65123)

[3] Elena Celledoni, Robert I. McLachlan, Brynjulf Owren, and G. R. W. Quispel, Energypreserving integrators and the structure of B-series, Found. Comput. Math. 10 (2010), no. 6, 673-693, DOI 10.1007/s10208-010-9073-1. MR2728426(2012d:65311) 
[4] E. Celledoni, B. Owren, and Y. Sun, The minimal stage, energy preserving RungeKutta method for polynomial Hamiltonian systems is the Averaged Vector Field method, arXiv:1203.3252.

[5] Philippe Chartier, Erwan Faou, and Ander Murua, An algebraic approach to invariant preserving integrators: the case of quadratic and Hamiltonian invariants, Numer. Math. 103 (2006), no. 4, 575-590, DOI 10.1007/s00211-006-0003-8. MR2221062(2007b:37209)

[6] Erwan Faou, Ernst Hairer, and Truong-Linh Pham, Energy conservation with nonsymplectic methods: examples and counter-examples, BIT 44 (2004), no. 4, 699-709, DOI 10.1007/s10543-004-5240-6. MR2211040 (2006k:37232)

[7] E. Hairer, Energy-preserving variant of collocation methods, J. Numer. Anal. Ind. Appl. Math. 5 (2010), no. 1-2, 73-84. MR2833602

[8] Ernst Hairer, Christian Lubich, and Gerhard Wanner, Geometric numerical integration, 2nd ed., Springer Series in Computational Mathematics, vol. 31, Springer-Verlag, Berlin, 2006. Structure-preserving algorithms for ordinary differential equations. MR2221614 (2006m:65006)

[9] E. Hairer and C.J. Zbinden, On conjugate-symplecticity of B-series integrators, IMA Journal of Numerical Analysis, 33 (2013), no. 1, 57-79. MR.3020950

[10] F. Iavernaro and D. Trigiante, High-order symmetric schemes for the energy conservation of polynomial Hamiltonian problems, J. Numer. Anal. Ind. Appl. Math. 4 (2009), no. 1-2, 87-101. MR.2598786(2011c:65291)

[11] Benedict Leimkuhler and Sebastian Reich, Simulating Hamiltonian dynamics, Cambridge Monographs on Applied and Computational Mathematics, vol. 14, Cambridge University Press, Cambridge, 2004. MR2132573 (2006a:37078)

[12] A. Murua, Formal series and numerical integrators. I. Systems of ODEs and symplectic integrators, Appl. Numer. Math. 29 (1999), no. 2, 221-251, DOI 10.1016/S0168-9274(98)00064-6. MR 1666537 (99k:65068)

[13] Matteo Petrera, Andreas Pfadler, and Yuri B. Suris, On integrability of HirotaKimura type discretizations, Regul. Chaotic Dyn. 16 (2011), no. 3-4, 245-289, DOI 10.1134/S1560354711030051. MR2810980 (2012f:37144)

[14] G. R. W. Quispel and D. I. McLaren, A new class of energy-preserving numerical integration methods, J. Phys. A 41 (2008), no. 4, 045206, 7, DOI 10.1088/1751-8113/41/4/045206. MR2451073 (2009j:65145)

[15] Ge Zhong and Jerrold E. Marsden, Lie-Poisson Hamilton-Jacobi theory and Lie-Poisson integrators, Phys. Lett. A 133 (1988), no. 3, 134-139, DOI 10.1016/0375-9601(88)90773-6. MR:967725(89h:58070)

Department of Mathematical Sciences, NTnU, N-7491 Trondheim, Norway

E-mail address: elenac@math.ntnu.no

Department of Mathematical Sciences, NTNU, N-7491 Trondheim, Norway

E-mail address: bryn@math.ntnu.no

LSEC, Institute of Computational Mathematics, Chinese Academy of Sciences (CAS),

P.O. Box 2719, BeiJing 100190, China

E-mail address: sunyj@lsec.cc.ac.cn 